\title{
Structure-function analysis of lipid substrates and inhibitors of sphingosine kinases
}

David R. Adams², Susan Pyne ${ }^{1^{*}}$ and Nigel J. Pyne ${ }^{1^{*}}$

${ }^{1}$ Strathclyde Institute of Pharmacy and Biomedical Sciences, University of Strathclyde, 161 Cathedral Street, Glasgow, G4 0RE Scotland, UK.

2 School of Engineering \& Physical Sciences, Heriot-Watt University, Edinburgh EH14 4AS, UK

* To whom correspondence should be addressed (‥j.pyne@strath.ac.uk)

Key words: Sphingosine 1-phosphate, sphingosine kinase, FTY720, (R)-FTY720 methyl ether, sphingadienes, safingol 


\section{Abstract}

The sphingosine kinases, SK1 and SK2, catalyse the formation of the bioactive signalling lipid, sphingosine 1-phosphate (S1P), from sphingosine. SK1 and SK2 differ in their subcellular localisation, trafficking and regulation, but the isoforms are also distinct in their selectivity towards naturally occurring and synthetic ligands as substrates and inhibitors. To date, only the structure of SK1 has been determined, and a structural basis for selectivity differences in substrate handling by SK2 has yet to be established. Here we present a structural rationale, based on homology modelling and ligand docking, to account for the capacity of SK2, but not SK1, to efficiently process the pharmacologically active substances, fingolimod (FTY720) and safingol, as substrates. We propose that two key residue differences in hSK2 (Ser305/Thr584 in place of Ala175/Ala339 in hSK1) facilitate conformational switching in the lipid head group anchor residue, Asp308 (corresponding to Asp178 in hSK1), to accommodate substrate diversity for SK2. Our analysis accounts for the contrasting behaviour of fingolimod and safingol as non-turnover inhibitors of SK1, but substrates for SK2, and the observed stereoselectivity for phosphorylation of the pro-S hydroxymethyl group of fingolimod to generate (S)-FTY720-P in vivo. We also rationalise why methylation of the pro-R hydroxymethyl of FTY720 switches the behaviour of the resulting compound, (R)-FTY720 methyl ether (ROMe), to SK2-selective inhibition. Whilst the pharmacological significance of (S)-FTY720-P is firmly established, as the active principle of fingolimod in treating relapsing-remitting multiple sclerosis, the potential importance of SK-mediated phosphorylation of other substrates, such as safingol and non-canonical naturally occuring substrates such as (4E,nZ)-sphingadienes, is less widely appreciated. Thus, the contribution of SK2-derived safingol 1-phosphate to the anti-cancer activity of safingol should be considered. Similarly, the biological role of sphingadiene 1phosphates derived from plant-based dietary sphingadienes, which we also show here are substrates for both SK1 and SK2, merits investigation. 


\section{Introduction}

Sphingosine 1-phosphate (S1P) is a bioactive lipid that regulates physiological effects through both receptor mediated signalling and intracellular target protein pathways. Two isoforms of sphingosine kinase (SK1 and SK2) catalyse the phosphorylation of sphingosine (Sph) to produce S1P (Fig. 1) [1]. These enzymes differ in their tissue expression, subcellular localisation and biochemical properties and regulate separate and overlapping cell biology [1]. S1P is degraded by irreversible cleavage at the $\mathrm{C}_{2}-\mathrm{C}_{3}$ bond to hexadecenal and phosphoethanolamine, catalysed by S1P lyase. S1P can also be dephosphorylated to sphingosine, catalysed by two isoforms of S1P phosphatase and non-specific lipid phosphate phosphatases. S1P promotes proliferation and survival, while ceramide induces apoptosis, growth arrest or senescence [1]. The balance between ceramide and S1P has been termed the 'sphingolipid rheostat' [2, 3] and involves receptor-mediated (autocrine, paracrine and signal amplification loops) and intracellular target protein-dependent effects of S1P that are generally functionally opposed by ceramide. At least one of the enzymes that is involved in the interconversion of ceramide, sphingosine and S1P is allosterically regulated. In this regard, S1P inhibits CerS2 by binding to two residues that comprise an S1P receptor-like motif found specifically in CerS2 [4]. This might therefore provide a mechanism that enables a rheostat to function in a reciprocal manner. Thus, inhibition of CerS2 by S1P might effectively bias the rheostat toward S1P synthesis. In addition, the inhibition of CerS2 by S1P might also reduce de novo synthesis of long chain ceramides by re-directing accumulated dihydrosphingosine toward dihydrosphingosine 1-phosphate formation [5]. However, there is additional complexity in the sphingolipid rheostat model as different molecular species of ceramide do not always promote cell death and, in some cases, can promote cell survival [6]. In addition, ceramide can be converted by ceramide kinase into ceramide 1-phosphate (C1P) [7], which is a pro-survival bioactive lipid. Nevertheless, the general consensus approach in pharmacologically-directed work is to develop new chemical entities that inhibit sphingosine kinases to restrict flux from ceramide to S1P and, thereby, to promote accumulation of apoptotic ceramides to deliver cytotoxic effects to, for instance, cancer cells.

Five differentially expressed $\mathrm{G}$ protein-coupled receptors (GPCRs), designated $\mathrm{S}_{1} \mathrm{P}_{1-5}$, mediate many of the physiological roles of S1P, such as trafficking of lymphocytes, regulation of vascular barrier integrity and modulation of vascular tone [1]. S1P receptors have been successfully targeted for therapeutic application. 
Thus, Gilenya ${ }^{\circledR}$ (a formulation of fingolimod/FTY720, Fig. 1) is used for treatment of relapsing and remitting multiple sclerosis. FTY720 is a sphingosine-like molecule that is phosphorylated by SK2 to form FTY720phosphate (FTY720-P), which is then exported from cells to agonise and induce proteasomal degradation of $\mathrm{S}_{1} \mathrm{P}_{1}$; termed functional antagonism [8]. This effectively abrogates $\mathrm{S}_{1} \mathrm{P}_{1}$-mediated $\mathrm{T}$-cell egress from lymph nodes and invasion of inflammatory T cells into the CNS. FTY720-P also reduces astrogliosis and promotes nerve re-myelination and recovery, thereby relieving symptoms of remitting multiple sclerosis. There is also abundant evidence for the involvement of S1P in cancer such as breast [9], ovarian [10], gastrointestinal [11], hepatocellular carcinoma [12] and glioblastoma [13]. Regardless of the cancer type, S1P is involved in tumour/stromal cell communication, the migration and invasiveness of cancer cells into the niche microenvironment, neovascularisation and metastasis. Indeed, signalling through S1P receptors contributes to, for example, signal amplification loops that drive oncogenesis. There is also an association between high S1P receptor and SK1 expression levels in tumours and poor clinical prognosis in terms of disease-specific survival and recurrence [14].

SK1 translocates from the cytoplasm to the plasma membrane in response to extracellular ligands (e.g. TNF $\alpha$ ) to access its substrate, Sph, while SK2 shuttles to and from the nucleus [15-17]. There are several intracellular targets of S1P. For instance, SK1-derived S1P binds to the RING domain of TNF receptor associated factor 2 (TRAF2), an E3 ligase which catalyses Lys63-polyubiquitination of RIP1, a protein kinase in the NF-кB pathway that regulates cell survival and inflammation [18]. S1P produced by SK2 also binds prohibitin 2 (PHB2) to regulate mitochondrial assembly and electron transport chain function at complex IV [19]. The catalytic subunit of telomerase, human telomerase reverse transcriptase (hTERT), is stabilised by binding S1P formed by SK2. This prevents interaction with the E3 ligase markorin ring finger protein (MKRN1), thereby abrogating ubiquitination of hTERT and its removal from cells by proteasomal degradation. The stabilisation of telomerase enhances proliferation and tumour growth [20]. S1P formed by nuclear SK2 inhibits HDAC1/2 to maintain lysine acetylation of histone [21] and to regulate peroxisome proliferator-activated receptor gamma (PPAR $\gamma$ ) to enhance gene expression [22]. Therefore, SK inhibitors have the potential to reduce inflammation, 
prevent replicative immortality and cell survival and alter mitochondrial function, gene expression and S1P receptor-mediated signalling in cancer.

There is a significant body of evidence demonstrating that the SK isoforms differ not only in their intracellular localisation, trafficking and regulatory behaviour, but that they exhibit distinct properties in their recognition of both natural and synthetic ligands as substrates. This article addresses a number of important unresolved questions concerning the binding of lipid substrates and inhibitors to SK1 and SK2. For example, what is the structural basis for isoform selectivity with highly flexible ligands-as with FTY720, a substrate for SK2 and an inhibitor of SK1, and (R)-FTY720 methyl ether (ROMe, Fig. 1), an SK2-selective inhibitor? What is the nature of the interaction of sphingosine kinases with 4,n-sphingadienes and safingol, lipids with anti-cancer properties, and why does the single centre stereochemistry change in the head group of safingol (Fig. 1) result in SK1-selective inhibition and SK2-selective substrate behaviour? Issues concerning substrate versus inhibitor activity are important, as they might fundamentally point to the need to re-evaluate the mechanism of action of these molecules as anti-cancer agents.

FTY720 is phosphorylated by SK2, yet reportedly functions as a competitive inhibitor of SK1 with a $K_{\mathrm{i}}$ of 2 $\mu \mathrm{M}[23,24]$. Although there is evidence that FTY720 is phosphorylated in Sphk2/- mice [25], suggesting that SK1 might also process FTY720 as a substrate, it is clear that it is, at best, a very poor substrate for SK1, and the possibility that an alternative kinase might function to catalyse the phosphorylation of FTY720 in Sphk2-mice has not been ruled out. Curiously the methyl ether derivative of FTY720, ROMe, fails to inhibit SK1 and is not a substrate for SK2 but rather inhibits this isoform [26]. As ROMe modulates endothelial barrier

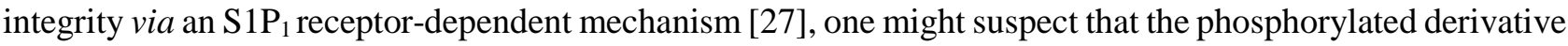
of ROMe could bind to the $\mathrm{S}_{1} \mathrm{P}_{1}$ receptor in a manner analogous to FTY720-P. However, neither SK1 nor SK2 catalyse phosphorylation of ROMe. Using structural analysis, we provide an explanation for these properties of SK1 and SK2 toward FTY720 and ROMe as substrate/inhibitors.

Doubly unsaturated sphingadienes, which occur naturally in plant-based sphingolipid dietary intake, are found in a wide range of mammalian tissues and are metabolized to ceramides and sphingadiene 1-phosphate with 
almost the same efficiency as sphingosine. Recently it has been reported that sphingadienes are substrates for SK1 and SK2, but that sphingadiene bases accumulate in the hippocampus of Sphk2 ${ }^{-/}$mice [28]. We have also found that $(4 E, \mathrm{nZ})$-sphingadienes $(\mathrm{n}=8,11,14)$ are substrates for both SK1 and SK2 (Fig. 2) using an established isofom specific radiometric assay [29]. However, they are less susceptible to degradation by the S1P lyase [30]. Fatty acid desaturase, FADS3 is a ceramide desaturase that is involved in the formation of sphingadienes [30]. Sphingadienes are bioactive and induce colon cancer cell death in vitro and prevent intestinal tumorigenesis in vivo [31]. This involves abrogated translocation of Akt from the cytosol to the plasma membrane, thereby inhibiting protein translation and promoting apoptosis and autophagy. Sphingadienes also reduce Wnt signalling, nuclear $\beta$-catenin localization, and levels of $\beta$-catenin and phosphorylated (inactive) GSK3 $\beta$ in colon cancer cells [32]. Indeed, inhibition of GSK3 $\beta$ attenuates the effects of sphingadienes. Sphingadienes also increase colonic S1P lyase expression and reduce S1P levels, STAT3 signalling, cytokine levels and tumorigenesis [33]. In addition, sphingadienes are cytotoxic to transformed and primary neuroblastoma cells, and this is independent of N-Myc amplification status and occurs via caspase-dependent apoptosis and autophagy [34]. However, the new evidence presented here and elsewhere, reporting that sphingadienes are substrates for SK1 and SK2, suggests that the biological effect of sphingadiene 1-phosphate on Akt signalling and GSK3 $\beta$ requires investigation. In addition, sphingadiene 1-phosphate might induce the up-regulation of S1P lyase expression, while the sphingadiene itself is likely to compete with sphingosine for the sphingosine binding site in SK. Both actions might therefore, account for the ability of sphingadienes to reduce S1P levels. Further investigation of the biological action of sphingadiene 1-phosphate is warranted.

Finally, we present evidence that safingol (L-threo-dihydrosphingosine), which is currently the only SK1 inhibitor to enter clinical trials for oncology, is a substrate for SK2 (Fig. 2). Safingol can be safely administered in combination with cisplatin, resulting in reduced S1P levels [35]. However, its ability to act as a substrate for SK2 is important because this suggests that L-threo-dihydrosphingosine 1-phosphate might also have anticancer activity. A structure-based rationale is set out for the behaviour of the D-erythro lipids, sphingosine and the sphingadienes, as substrates for both SK1 and SK2, and the switch to SK1-inhibitory/SK2-substrate behaviour with the L-threo head group of safingol. 


\section{Structural features of the sphingosine and ATP binding sites in sphingosine kinases}

The current position with regards to SK1 structural characterisation has been reviewed recently and key structure-function hypotheses relating to mechanism of action and regulatory control set out [36]. In contrast to SK1, there are no crystal structures for SK2 available at present, and the structural basis for subtle differences in substrate selectivity preferences between SK1 and SK2 has received little attention to date. These differences between SK1 and SK2 may be relevant to the design of isoform-selective inhibitors, but they also have wider physiological and pharmacological implications. Thus, FTY720 (fingolimod, Gilenya ${ }^{\circledR}$ ), an immunomodulatory prodrug used for treatment of multiple sclerosis [37], is accommodated as a substrate by SK2 and converted in vivo into the active phosphorylated form of the drug, (S)-FTY720-P (Fig. 1), which serves as an S1P receptor agonist [38] that functionally antagonises S1P signalling by inducing internalisation

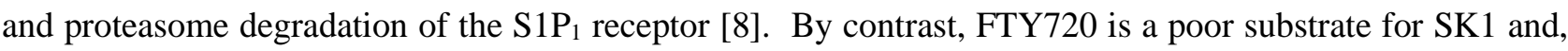
therefore, binds as an inhibitor to this isoform with only very weak, if any, turnover to phosphorylated product [23]. Intriguingly, methylation of the pro- $R$ hydroxymethyl group of fingolimod, to generate (R)-FTY720 methyl ether (ROMe) (Fig. 1), switches the compound properties to SK2-selective inhibition [26]. Here we propose a rationale for these observations based on analysis of an SK1-derived homology model for SK2 [39].

SK1 exhibits a bi-domain structure comprising an N-terminal domain (NTD) that binds the nucleotide cosubstrate (ATP) and a C-terminal domain (CTD) that hosts the lipid substrate binding site (Fig. 3A). The CTD is formed from a $\beta$-sandwich core with three lipid binding loops (LBL-1, LBL-2 and LBL-3) packing on one face of the $\beta$-sandwich to generate a curved hydrophobic cavity, the 'J-channel', that accommodates the lipid substrate tail. The reverse face of the CTD $\beta$-sandwich is occupied by a single long regulatory loop (the 'Rloop') that hosts a phosphorylation site for ERK kinase. The phosphorylation of this site in SK1 provides a mechanism for targeting and activation of S1P production at the plasma membrane [40, 41]. In SK2 the Rloop is greatly extended and additionally contains a nuclear export signal sequence that is regulated through phosphorylation by PKD [17, 42]. This, together with an extended $\mathrm{N}$-terminal sequence that contains a nuclear localisation signal sequence [17], is a key distinguishing feature for SK2 and contributes to a distinctive pattern of subcellular localisation and trafficking as compared to SK1 [43]. The core NTD of SK1 and SK2 exhibits 
strong homology, however, with sequence conservation particularly high in four key regions that engage bound nucleotide (Figure S1 in Adams et al. [36]). The nucleotide binds with an accompanying $\mathrm{Mg}^{2+}$ ion that forms the catalytic centre at the NTD/CTD interface, and a DGE-containing hairpin, contributed by the CTD $\beta$ sandwich (between $\beta 14 / \beta 15$ in SK1), plays an important role in coordinating the metal ion. The head group of bound lipid substrate is trapped between this motif (341-DGE-343 in human SK1, hSK1) and lipid binding loops LBL-1 and LBL-2 at the mouth of the J-channel, and the DGE hairpin plays an important role in orchestrating presentation of the head group for phosphorylation.

Current understanding of ligand binding is informed by five hSK1 co-crystal structures that exhibit J-channel occupancy either with bound sphingosine (Sph) (PDB: 3VZB, [44]) or an inhibitor. To date, structure determination has been accomplished with three inhibitors, namely with SKi (3VZC, [44]), Amgen-23 (4L02, [45]) and PF-543 (4V24, [46]) (Fig. 1); in the case of SKi the structure has additionally been determined with bound Mg-ADP (3VZD, [44]). The Sph co-crystal structure (3VZB) reveals that the substrate head group is engaged by a highly organised polar network that includes Asp178 on LBL-1 as a hydrogen bonding anchor for the Sph 3-OH (Fig. 3B, C). The hydroxyl is also hydrogen bonded to a structural water (W1) that is networked to Ala339 and Gly342 on the DGE hairpin and to the side chain of Ser168, another residue contributed by the CTD $\beta$-sandwich core ( $\beta 8)$. The side chain of Ser168 additionally serves as a hydrogen bond acceptor for the Sph ammonium ion. Two slightly differing positions are observed for the Sph 1-OH in different protein chains of the 3VZB crystal structure, one with a hydrogen bond contact to the backbone carbonyl of LBL-2 residue Leu268 and one with a contact to Asp81. The latter is presented by the sphingosine kinase 'P-loop' and is thought to serve as the base for deprotonation of the phosphoacceptor hydroxyl group during substrate phosphorylation. The P-loop is the sequence running from $\beta 3$ into $\alpha 3$ in the SK1 NTD, and it also serves to bind the nucleotide's $\alpha$-phosphate. In addition to engaging the substrate 1-OH, the Asp81 side chain carboxylate is positioned so as to cap the N-terminus of helix- $\alpha 5$, which, together with the preceding residues issuing from $\beta 4$, form the conserved sphingosine kinase 'T-loop'. The T-loop binds the nucleotide's $\beta$-phosphate, and it is postulated that plasticity in the backbone of this loop and in the presentation Asp81 may be important for catalytic turnover [36]. Although no co-crystal structures are currently available with ATP (or a non-hydrolysable triphosphate mimetic), it is clear from the crystal structure (3VZD) [44] with bound 
ADP that the ATP $\gamma$-phosphate would be positioned proximal to the Sph 1-OH group when hydrogen bonded to Asp81.

The structural water (W1) is conserved across all of the SK1 crystal structures, irrespective of ligand occupancy in the J-channel. In the case of the SKi co-crystal structures, the ligand sits deep in the J-channel as a Sphcompetitive inhibitor but lacks an extended Sph-mimetic head group. In this instance, the phenolic group of SKi hydrogen bonds to one oxygen of the Asp178 carboxylate whilst the second oxygen is engaged by an additional water molecule (W2) that occupies the position of the Sph 3-OH hydroxyl and completes the network to W1 (Fig. 4A). Partial occupancy by a third water molecule (W3) at the position of the Sph-1-OH is also seen in the SKi co-crystal structures. The surrogacy of W2 and W3 for the Sph 3-OH and 1-OH in the SKi co-crystal structures highlights that these sites are favourable hydroxyl binding loci. Amgen-23 conserves the core structure of SKi but replaces the phenol by a hydroxylated basic piperidine-containing head group. In this case, the hydroxymethyl side chain maps to the position of Sph 3-OH/W2 whilst the secondary carbinol centre occupies the position of the Sph 1-OH/W3 and engages Asp81 (Fig. 4B). The protonated piperidine nitrogen is perfectly positioned to target Asp178, so that one carboxylate oxygen is engaged by the piperidinium centre and the second with a hydrogen bond from the hydroxymethyl group. The J-channel surface is overwhelmingly hydrophobic but does present an exposed threonine (Thr196, conserved as Thr326 in $h$ SK2) as a potential hydrogen bonding point, and the crystal structures reveal that the aminothiazole subunit in both SKi and Amgen-23 exploits this contact. PF-543 does not hydrogen bond to the J-channel threonine but does engage the residues at the mouth of the J-channel in a similar manner to Amgen-23. Thus, the cocrystal structure [46] reveals that the hydroxymethylpyrrolidine head group of PF-543 gives a strong double contact on the Asp178 carboxylate from the protonated pyrrolidine nitrogen and hydroxyl group, the latter mapping to the Sph 3-OH/W2 position (Fig. 4C). The ring nitrogen atoms of PF-543 and Amgen-23 exhibit high positional correspondence to one another, and point to a favourable ammonium ion binding locus beneath the SK1 Asp178 residue that differs from the position occupied by the substrate primary ammonium centre in the Sph co-crystal structure. The combination of a cyclic tertiary ammonium ion with a $\beta$-hydroxy group from a hydroxymethyl side chain provides a geometrically optimised ligand combination for tight end-on engagement of the Asp178 carboxylate. 
The direct ligand contact surface of the J-channel comprises some 20 residues. Of these, the $h \mathrm{SK} 1$ and SK2 isoforms differ only at three points, namely in Ile174, Met272, and Phe288 of SK1, corresponding respectively to Val304, Leu517, and Cys533 in SK2 (marked in the Fig. 4 schematics). Ile174 and Met272 contribute to the throat of the J-channel, whilst Phe288 stoppers the toe. Of these substitutions, the replacement of Phe288 by Cys533 in SK2 is predicted to have the greatest impact and is expected to result in a longer J-channel and potentially also to confer greater surface plasticity in SK2 due to loosened packing against adjacent hydrophobic residues. Another important contact area for ligands in the J-channel is at the heel, where the surface is contributed by an isoleucine located on LBL-3 (Ile302 in SK1, marked in the Fig. 4 schematics). Although this residue is conserved in SK2 (as Ile547), analysis of adjacent (non-contact surface) residue differences in LBL-3, together with consideration of the packing of LBL-3 against LBL-2, suggested that the heel isoleucine in SK2 may potentially experience an inward shift relative to that of SK1. This led to a hypothesis that the SK2 J-channel may possess an inwardly contracted heel compared to SK1 [39]. The SK1/PF-543 co-crystal structure revealed that the tail sulfonyl group of PF-543 lies proximal to the J-channel heel residue. Thus, modification of the inhibitor structure to reduce steric demand at this point whilst simultaneously expanding demand in the toe region of the J-channel would be expected to favour binding to SK2 and discriminate against SK1. These ideas were recently validated with the design of HWG-35D (compound 55 in Adams et al. [39]) as an SK2-selective inhibitor (Fig. 1 and Fig. 4D), where a switch from the potent SK1-selective inhibition of PF-543 (SK1 IC 5028 nM, > 100-fold selectivity over SK2) to nanomolar potent selective inhibition of SK2 was observed (SK2 IC $5041 \mathrm{nM}$, with 100-fold selectivity over SK1). This reversal in behaviour therefore corresponds to a selectivity swing of $>10,000$ fold between the structurally related PF-543 and HWG-35D inhibitors.

Although the heel and toe differences between SK1 and SK2 are likely the major determinants underlying the remarkable inhibitory selectivity switch between PF-543 and HWG-35D, there are other residue differences between $h \mathrm{SK} 1$ and $h \mathrm{SK} 2$ that may be relevant to the selectivity profile of other inhibitor series and that may also play a key role in dictating differences in substrate selectivity. In this regard, two important residue differences between the isoforms are likely to be Ala175 and Ala339 in hSK1 (marked in Fig. 3B), corresponding to Ser305 and Thr584 in hSK2. These two residues are located, respectively, on LBL-1 and on 
$\beta 14$ (just preceding the DGE hairpin); jointly these substitutions will impact on the packing interface between LBL-1 and the CTD $\beta$-sandwich core and may affect the side chain conformational properties for Asp178 (Asp308 in SK2), the key anchor residue for the Sph 3-OH group (Fig. 3B, C).

Slight positional movement is seen in the tip of LBL-1 across the currently available SK1 crystal structures. This may be due to differences in crystal lattice packing in the solid state but could also reflect inherent flexibility in LBL-1, which is postulated to dynamically open and close in order to extract Sph substrate from the membrane [36]. Nevertheless, a common conformation is seen for the Asp178 side chain across all of the SK1 crystal structures, with only minor rotational differences in the terminal carboxylate about the $\mathrm{C}_{\beta}-\mathrm{C}_{\gamma}$ bond. Inspection of the crystal structures suggests that the conformation adopted for the $\mathrm{C}_{\alpha}-\mathrm{C}_{\beta}$ torsion of Asp178 in SK1 may be constrained by the close packing of LBL-1 against the DGE hairpin, the latter exerting both steric constraints and charge-dipole opposition from the Gly342 backbone carbonyl to limit conformational freedom.

\section{FTY720 and (R)-FTY720 methyl ether (ROMe) as inhibitors and substrates of sphingosine kinases}

In considering the interaction of FTY720 with SK1, it would be tempting to map the primary ammonium ion and pro-S hydroxymethyl of FTY720 (see designation in Fig. 1) directly onto the corresponding features of Sph, as seen in the Sph/SK1 co-crystal structure (Fig. 3B, C). However, such a binding mode would orientate the pro- $R$ hydroxymethyl branch, which is absent in Sph, into a position of severe steric interdiction with the backbone of Leu268 (marked in Fig. 3B). Docking of FTY720 to SK1 (Fig. 5A) suggests that an alternative arrangement for the head group might be adopted, however, in which the primary ammonium centre and pro$R$ hydroxymethyl simultaneously engage Asp178, whilst the pro-S hydroxymethyl is favourably hydrogen bonded to Asp81 and, therefore, potentially disposed for phosphorylation to generate (S)-FTY720-P. In this arrangement the pro- $R$ hydroxymethyl oxygen would correspond well to the crystallographically defined position of the Sph 3-OH and the hydroxymethyl oxygen centres of both Amgen-23 and PF-543, hydrogen bonding favourably both to Asp178 and structural water W1. The primary ammonium ion placement for FTY720 in this model (Fig. 5A) approximates to the position occupied by the tertiary ammonium centres of Amgen-23 and PF-543 (Fig. 5A box). However, the nature of the branching in FTY720 in this arrangement is 
such that the head group fit between Asp178 and the opposing LBL-2 backbone is rather compressive, and the ammonium ion is also somewhat out of position for a geometrically optimised interaction with the Asp178 carboxylate. Moreover, adoption of this binding mode requires a near eclipsing arrangement in the bond from the FTY720 C4 centre to the aromatic ring. An alternative SK1-docked binding mode for FTY720 is illustrated in Fig. 5B. In this alternative state the C4-arene bond of FTY720 is rotated into a more favourable torsional arrangement whilst essentially maintaining the plane and position of the arene within the J-channel throat. A concomitant rotation of the polar head group is required to accommodate this backbone arrangement for FTY720, however, and this moves the pro-S hydroxymethyl out of contact with Asp81 and into engagement with Asp178 instead. The pro- $R$ hydroxymethyl is orientated into a hydrogen bonding position on one side of the carbonyl group of Leu268, whilst the primary ammonium occupies a hydrogen bonding position on the other side of the carbonyl, with salt bridging potential to Asp81. This analysis is consistent with the known properties of FTY720 as an inhibitor of SK1 that is only very weakly turned over, if at all, as a substrate, the latter conceivably occurring via reorganisation of the head group torsions to switch from the inhibitory binding mode of Fig. 5B to the potentially less favourable mode of Fig. 5A that presents the pro-S hydroxymethyl to Asp81 at the catalytic centre. In the proposed 'non-turnover' binding state there is potential for bridging water occupancy (not shown in Fig. 5B) between one of Asp178 carboxylate oxygens and W1 (analogous to W2 surrogacy in the SKi co-crystal structure) and also between the ammonium ion and Ser168, although such bridging waters would require displacement in order to switch into the proposed turnover state binding mode of Fig. 5A.

In the case of SK2, we postulate that the replacement of Ala175 and Ala339 in hSK1 by Ser305 and Thr584 in $h \mathrm{SK} 2$ allows access to an alternative rotameric state for the head group-anchoring aspartate in LBL-1 (Asp308). Two considerations underpin this hypothesis: firstly, the bulky nature of Thr584 (as compared to Ala339 in hSK1) is likely to drive some separation between the DGE-hairpin and the Asp308 side chain, thereby reducing steric constraints on rotation for the latter; and secondly, Thr584 may actively support an alternative rotameric state by hydrogen bonding to one of the Asp308 carboxylate oxygens. Moreover, in the favoured rotameric state for the Thr584 side chain, a hydrogen donor/acceptor relay may be possible from Ser305 to Thr584 and then from Thr584 to Asp308 (Fig. 5C). The combination of these effects may be 
sufficient to allow switching of the Asp308 side chain between an SK1-like state (orientated towards Leu268) and a 'lifted state' (orientated towards the DGE hairpin). This latter state would generate additional space between LBL-2 and Asp308 for accommodation of a branched substrate such as FTY720. Indeed, docking of FTY720 to the SK2 model in this state reveals that the ligand may be comfortably accommodated with a noncompressive salt bridge between its primary ammonium centre and Asp308, whilst the pro- $R$ hydroxymethyl group is hydrogen bonded to structural water W1 (assumed to be conserved in SK2) and the pro-S hydroxymethyl engages the catalytic base, Asp211, in a position well suited for phosphorylation to generate (S)-FTY720-P (Fig. 5C). The plane and position of the arene in the throat of the J-channel maps almost perfectly onto that of PF-543, as seen in the co-crystal structure of the latter with SK1 [46]. In contrast to the FTY720/SK1 model of Fig. 5A, the ligand backbone arrangement in the FTY720/SK2 model is relaxed and lacks the unfavourable eclipsing torsion of the former.

ROMe is an SK2-selective inhibitor $[24,26]$ that is formally derived from FTY720 by methylation of the pro$R$ hydroxymethyl group. In the light of the preferred inhibitory binding mode postulated for FTY720 in Fig. 5B, it is fully reasonable to expect a loss of binding affinity for ROMe with SK1. Thus, the tight hydrogen bond invoked in this model between the pro- $R$ hydroxymethyl and Leu268 will be lost for ROMe and the methyl ether will sterically obstruct binding to SK1 with such a head group orientation. However, with an alternative rotameric state accessible to Asp308 in SK2, ROMe docks to the J-channel with the ammonium ion comfortably accommodated beneath the aspartate and with the remaining hydroxymethyl branch of the head group now hydrogen bonded to the LBL-2 leucine carbonyl (Leu513, corresponding to Leu268 in SK1) (Fig. 5D). The methoxymethyl ether fits tidily into a subpocket between Leu513 and a second leucine (Leu297) located on $\beta 8$. Moreover, in this orientation the ether oxygen is perfectly positioned to interact with a bridging water molecule hydrogen bonded to the catalytic base at the W3 position seen in the SK1/SKi co-crystal structures (schematised in Fig. 4A). The aromatic ring of ROMe sits a little deeper in the SK2 J-channel than that predicted for docked FTY720 (Fig. 5C) and maps closely onto the crystallographically defined position of the $p$-phenylene subunit for Amgen-23 bound to SK1 [45] (Fig. 5D box). 
The selectivity of ROMe for inhibition of $h S K 2$ over $h S K 1$ is conserved for the murine SK isoforms ( $m S K 1$ and $m$ SK2). This is noteworthy because the $h$ SK2 ligand contact residues in the J-channel throat that differ from $h$ SK1 (Val304 and Leu517, corresponding to Ile174 and Met272 in hSK1) are found in mSK1 (as Val174 and Leu271). In the $h$ SK2/ROMe model these residues (not explicitly shown in Fig. 5D) are predicted to flank the inhibitor's $p$-phenylene subunit. The transposition of the hSK2 throat residues into mSK1 suggests, therefore, that the subtle difference in residue identity at these two positions is not a major determinant of isoform selectivity for ROMe. Indeed, cross-species sequence alignment comparisons reveal a degree of variability in the identity of these two J-channel throat residues, although they are universally hydrophobic in character for both SK1 and SK2 isoforms. In contrast, the combination of serine and threonine at the LBL-1/ $\beta$ sandwich interface (Ser305 and Thr584 of hSK2) appears to be absolutely conserved across species, consistent with an evolutionary significance to this distinguishing residue combination for SK2.

\section{Sphingadienes and safingol as substrates for sphingosine kinase}

Although the J-channel surface differs between $h \mathrm{SK} 1$ and hSK2 in the heel and toe regions (vide supra, discussion of HWG-35D), these differences are unlikely to have great impact on the binding of ligands with highly flexible $n$-alkyl chain tails such as FTY720, ROMe and endogenous substrates. For such compounds, the tails exhibit high conformational flexibility and are likely to be able to adapt readily to the distinctive contouring of the hydrophobic J-channel surface in the foot of SK1 and SK2. This notion is supported by comparison of the sphingadienes (Fig. 1) as substrates for the two isoforms, where the presence of additional $\Delta^{8,9}, \Delta^{11,12}$ or $\Delta^{14,15}$ geometrically constrained unsaturation has little or no impact on the capacity of these compounds to serve as substrates for SK1 and SK2 (Fig. 2). Indeed, docking of these sphingadienes, illustrated for SK1 in Fig. 6A, reveals that they can be comfortably accommodated in the J-channel with the same binding mode for the D-erythro head group as seen in the co-crystal structure of Sph itself (3VZB) [44]. The $\Delta^{8,9}$ double bond of 4,8-sphingadiene is predicted to occupy a position and plane close to that of the $p$-phenylene ring of Amgen-23. For 4,11-sphingadiene and 4,14-sphingadiene, the $\Delta^{11,12}$ and $\Delta^{14,15}$ double bonds map, respectively, to the thiazole and terminal phenyl rings of Amgen-23 and SKi. 
It is likely that SK1 is more discriminating in regard to substrate head group stereochemistry than SK2. Thus, safingol (L-threo-sphinganine; Fig. 1), an SK1- and PKC-inhibitory compound with anti-cancer activity [35, 47-50] embodies a switch in C-3 stereochemistry that appears to compromise the capacity of this compound to serve as a substrate for SK1, whereas appreciable turnover is observed with SK2 (Fig. 2). In the case of SK1, binding of the ammonium ion and primary alcohol in the D-erythro substrate positions, as for Sph and the sphingadienes just discussed, would require the epimeric carbinol of safingol to be unfavourably compressed into the side chain of Leu268. Consistent with this, safingol docks into the SK1 structure with an alternative head group arrangement that provides escape from this compression. According to our model (Fig. 6B), the L-threo head group is orientated so as maintain engagement of the 3-OH with Asp178 and bring the ammonium ion into contact with Asp81. The hydroxymethyl group is thence rotated out of contact with the catalytic base and is instead locked down with a hydrogen bond to the carbonyl of Leu268. However, there is likely a small amount of residence time where the ligand bond rotations put the head group of safingol into a Sph-like arrangement for turnover, even though it is a less favourable organisation due to the loss of the hydrogen bond from the 3-OH to Asp178 and with some compression of the 3-OH on Leu268. This would account for the profile of safingol as an inhibitor of SK1 with substrate processing, although very weak, nevertheless just detectable in the SK1 assay with $50 \mu \mathrm{M}$ safingol (Fig. 2). Therefore, the predominance is for safingol to adopt a 'non-turnover' state in SK1 (Fig. 6B). In contrast, safingol docks to SK2 so as to conserve the contact between the hydroxymethyl and Asp211 catalytic base (Fig. 6C). This turnover-competent binding mode is again dependent on the ability of the LBL-1 head group anchor residue (Asp308) to access the alternative 'lifted' rotameric state, which can then accommodate the safingol ammonium ion in direct salt bridge contact.

\section{Conclusion}

The marked selectivity of FTY720 and safingol as substrates for SK2 over SK1 suggests that SK2 may be more tolerant of changes in head group structure than SK1. This tendency for SK2 to be more accommodating in regard to the structure of ligands accepted as substrates has also been demonstrated in studies with a wider range of analogues of FTY720 [51]. We propose that this characteristic of SK2, which is essential for the 
pharmacological activity of FTY720, is related to the ability of the LBL-1 anchor aspartate to access conformational space that is restricted in SK1. Isoform-specific differences in the interface between LBL-1 and the DGE hairpin likely underpin this distinctive characteristic, and the replacement of Ala339 in SK1 by Thr584 on the DGE hairpin of SK2, possibly in combination with Ser305 (in place of Ala175) on LBL-1, may be central to this distinguishing behaviour.

From a wider perspective, in terms of the biological effects of dietary sphingadienes and safingol, it will be important to establish to what extent these are due to the compounds themselves or to the phosphorylated derivatives of the parent compounds. If the latter, then biotransformation could be a point of exploitation as, for instance, the expression levels of both SK1 and SK2 are increased in various cancers, and this might therefore advantageously confer cancer-specific targeting. It will also be important to establish in detail which signalling networks are affected by the sphingadiene 1-phosphates and by safingol 1-phosphate. Thus, the effects of sphingadienes on Wnt/GSK3 $\beta /$ Akt/STAT3 signalling, for example, might be due to the phosphorylated equivalent, possibly by antagonising S1P receptors and/or by competing with S1P for binding to target effectors regulating transcriptional/translational programmes in cancer. Moreover, 4,14-sphingadiene is naturally occurring in the brain, and therefore, the physiological role in homeostatic regulation of S1Pdependent signalling should be further investigated.

\section{Acknoledgements}

We thank Avanti Polar Lipids (USA) for the kind gift of the (4E,nZ)-Sphingadienes and Professor A.H. Merrill Jr (Georgia Institute of Technology, USA) for the gift of safingol.

\section{References}

1. Pyne S, Adams DR, Pyne, NJ (2016) Sphingosine 1-phosphate and sphingosine kinases in health and disease: Recent advances. Prog Lipid Res 62:93-106. 
2. Pyne S, Chapman J, Steele L, Pyne NJ (1996) Sphingomyelin-derived lipids differentially regulate the extracellular signal-regulated kinase 2 (ERK-2) and c-Jun N-terminal kinase (JNK) signal cascades in airway smooth muscle. Eur J Biochem 237:819-82.

3. Cuvillier O, Pirianov G, Kleuser B, Vanek PG, Coso OA, Gutkind S, Spiegel S (1996) Suppression of ceramide-mediated programmed cell death by sphingosine-1-phosphate. Nature 381:800-803.

4. $\quad$ Laviad EL, Albee L, Pankova-Kholmyansky I, Epstein S, Park H, Merrill AH Jr, Futerman AH (2008) Characterization of ceramide synthase 2: tissue distribution, substrate specificity, and inhibition by sphingosine 1-phosphate. J Biol Chem 283:5677-5684.

5. Siow D, Sunkara M, Morris A, Wattenberg B (2015) Regulation of de novo sphingolipid biosynthesis by the ORMDL proteins and sphingosine kinase-1. Adv Biol Regul 57:42-54.

6. Saddoughi SA, Ogretmen B (2013) Diverse functions of ceramide in cancer cell death and proliferation. Adv Cancer Res 117:37-58.

7. Hoeferlin LA, Wijesinghe DS, Chalfant CE (2013) The role of ceramide-1-phosphate in biological functions. Handbook Exp Pharmacol 215:153-166.

8. Brinkmann V, Cyster JG, Hla T (2004) FTY720: sphingosine 1-phosphate receptor-1 in the control of lymphocyte egress and endothelial barrier function. American journal of transplantation: official journal of the American Society of Transplantation and the American Society of Transplant Surgeons 4:1019-1025.

9. $\quad$ Singh SK, Spiegel S (2020) Sphingosine-1-phosphate signalling: A novel target for simultaneous adjuvant treatment of triple negative breast cancer and chemotherapy-induced neuropathic pain. Adv Biol Regul 75:100670.

10. Hernández-Coronado CG, Guzmán A, Castillo-Juárez H, Zamora-Gutiérrez D, Rosales-Torres AM (2019) Sphingosine-1-phosphate (S1P) in ovarian physiology and disease. Annales d'endocrinologie 80:263272.

11. Sukocheva OA, Furuya H, Ng, ML, Friedemann M, Menschikowski M, Tarasov VV, Chubarev VN, Klochkov SG, Neganova ME, Mangoni AA, Aliev G, Bishayee A (2020) Sphingosine kinase and sphingosine- 
1-phosphate receptor signalling pathway in inflammatory gastrointestinal disease and cancers: A novel therapeutic target. Pharmacol Therap 207:107464.

12. Maceyka, M., Rohrbach, T., Milstien, S., \& Spiegel, S (2020) Role of Sphingosine Kinase 1 and Sphingosine-1-Phosphate Axis in Hepatocellular Carcinoma. Handbook Exp Pharmacol 259:3-17.

13. Tea MN, Poonnoose SI, Pitson SM (2020) Targeting the Sphingolipid System as a Therapeutic Direction for Glioblastoma. Cancers 12:111.

14. Watson C, Long JS, Orange C, Tannahill CL, Mallon E, McGlynn LM, Pyne S, Pyne NJ, Edwards J (2010) High expression of sphingosine 1-phosphate receptors, S1P1 and S1P3, sphingosine kinase 1, and extracellular signal-regulated kinase-1/2 is associated with development of tamoxifen resistance in estrogen receptor-positive breast cancer patients. The Am J Pathol 177:2205-2215.

15. Jarman KE, Moretti PA, Zebol JR, Pitson SM (2010) Translocation of sphingosine kinase 1 to the plasma membrane is mediated by calcium- and integrin-binding protein 1. J Biol Chem 285:483-492.

16. Maceyka M, Sankala H, Hait NC, Le Stunff H, Liu H, Toman R, Collier C, Zhang M, Satin LS, Merrill AH, Jr, Milstien S, Spiegel S (2005). SphK1 and SphK2, sphingosine kinase isoenzymes with opposing functions in sphingolipid metabolism. J Biol Chem 280:37118-37129.

17. Ding G, Sonoda H, Yu H, Kajimoto T, Goparaju SK, Jahangeer S, Okada T, Nakamura S (2007) Protein kinase D-mediated phosphorylation and nuclear export of sphingosine kinase 2. The J Biol Chem 282:27493-27502.

18. Alvarez SE, Harikumar KB, Hait NC, Allegood J, Strub GM, Kim EY, Maceyka M, Jiang H, Luo C, Kordula T, Milstien S, Spiegel S (2010) Sphingosine-1-phosphate is a missing cofactor for the E3 ubiquitin ligase TRAF2. Nature 465:1084-1088.

19. Strub GM, Paillard M, Liang J, Gomez L, Allegood JC, Hait NC, Maceyka M, Price MM, Chen Q, Simpson DC, Kordula T, Milstien S, Lesnefsky EJ, Spiegel S (2011) Sphingosine-1-phosphate produced by sphingosine kinase 2 in mitochondria interacts with prohibitin 2 to regulate complex IV assembly and respiration. FASEB journal: official publication of the Fed Am Soc Exp Biol 25:600-612. 
20. Panneer Selvam S, De Palma RM, Oaks JJ, Oleinik N, Peterson YK, Stahelin RV, Skordalakes E, Ponnusamy S, Garrett-Mayer E, Smith CD, Ogretmen B. (2015) Binding of the sphingolipid S1P to hTERT stabilizes telomerase at the nuclear periphery by allosterically mimicking protein phosphorylation. Science Signal 8:ra58.

21. Hait NC, Allegood J, Maceyka M, Strub GM, Harikumar KB, Singh SK, Luo C, Marmorstein R, Kordula T, Milstien S, Spiegel S (2009) Regulation of histone acetylation in the nucleus by sphingosine-1phosphate. Science 325:1254-1257.

22. Parham KA, Zebol JR, Tooley KL, Sun WY, Moldenhauer LM, Cockshell MP, Gliddon BL, Moretti PA, Tigyi G, Pitson SM, Bonder CS (2015) Sphingosine 1-phosphate is a ligand for peroxisome proliferatoractivated receptor- $\gamma$ that regulates neoangiogenesis. FASEB journal: official publication of the FASEB J 29:3638-3653.

23. Tonelli F, Lim KG, Loveridge C, Long J, Pitson SM, Tigyi G, Bittman R, Pyne S, Pyne NJ (2010) FTY720 and (S)-FTY720 vinylphosphonate inhibit sphingosine kinase 1 and promote its proteasomal degradation in human pulmonary artery smooth muscle, breast cancer and androgen-independent prostate cancer cells. Cell Signal 22:1536-1542.

24. Lim KG, Tonelli F, Li Z, Lu X, Bittman R, Pyne S, Pyne NJ (2011) FTY720 analogues as sphingosine kinase 1 inhibitors: enzyme inhibition kinetics, allosterism, proteasomal degradation, and actin rearrangement in MCF-7 breast cancer cells. J Biol Chem 286:18633-18640.

25. Liang J, Nagahashi M, Kim EY, Harikumar KB, Yamada A, Huang W-H, Hait NC, Allegood JC, Price MM, Avni D, Takabe K, Kordula T, Milstien S, Spiegel S (2013) Sphingosine-1-phosphate links persistent STAT3 activation, chronic intestinal inflammation, and development of colitis-associated cancer. Cancer Cell 23:107-120.

26. Lim KG, Sun C, Bittman R, Pyne NJ, Pyne S (2011) (R)-FTY720 methyl ether is a specific sphingosine kinase 2 inhibitor: Effect on sphingosine kinase 2 expression in HEK 293 cells and actin rearrangement and survival of MCF-7 breast cancer cells. Cell Signal 23:1590-1595. 
27. Camp SM, Chiang ET, Sun C, Usatyuk PV, Bittman R, Natarajan V, Garcia JGN, Dudek SM (2016) Pulmonary Endothelial Cell Barrier Enhancement by Novel FTY720 Analogs: Methoxy-FTY720, FluoroFTY720, and $\beta$-Glucuronide-FTY720 . Chem Phys Lipids. 194:85-93.

28. Couttas TA, Rustam YH, Song H, Qi Y, Teo, JD, Chen J, Reid GE, Don AS (2020) A Novel Function of Sphingosine Kinase 2 in the Metabolism of Sphinga-4,14-Diene Lipids. Metabolites.10:236.

29. Baek DJ, MacRitchie N, Anthony N, Mackay SP, Pyne S, Pyne NJ, Bittman R (2013) structure activity relationships and molecular modeling of sphingosine kinase inhibitors. J Med Chem 56: 9310-9327.

30. Jojima K, Edagawa M, Sawai M, Ohno Y, Kihara A (2020) Biosynthesis of the anti-lipid-microdomain sphingoid base 4,14-sphingadiene by the ceramide desaturase FADS3. FASEB J 34:3318-3335.

31. Fyrst H, Oskouian B, Bandhuvula P, Gong Y, Byun H-S, Bittman R, Lee AR, Saba JD (2009) Natural sphingadienes inhibit Akt-dependent signaling and prevent intestinal tumorigenesis. Cancer Res 69:94579464.

32. Kumar A, Pandurangan AK, Lu F, Fyrst H, Zhang M, Byun H-S, Bittman R, Saba JD (2012) Chemopreventive sphingadienes downregulate Wnt signaling via a PP2A/Akt/GSK3 $\beta$ pathway in colon cancer. Carcinogenesis. 33:1726-1735.

33. Degagné E, Pandurangan A, Bandhuvula P, Kumar A, Eltanawy A, Zhang M, Yoshinaga Y, Nefedov M, de Jong PJ, Fong LG, Young SG, Bittman R, Ahmedi Y, Saba JD (2014) Sphingosine-1-phosphate lyase downregulation promotes colon carcinogenesis through STAT3-activated microRNAs. J Clin Invest 124:53685384.

34. Zhao P, Aguilar AE, Lee JY, Paul LA, Suh JH, Puri L, Zhang M, Beckstead J, Witkowski A, O Ryan R, Saba JD (2018) Sphingadienes show therapeutic efficacy in neuroblastoma in vitro and in vivo by targeting the AKT signaling pathway. Invest New Drugs 36:743-754.

35. Dickson MA, Carvajal RD, Merrill AH Jr, Gonen M, Cane LM, Schwartz GK. (2011) A phase I clinical trial of safingol in combination with cisplatin in advanced solid tumors. Clin Cancer Res 17:24842492. 
36. Adams DR, Pyne S, Pyne NJ (2016) Sphingosine Kinases: Emerging Structure-Function Insights. Trends Biochem Sci 41:395-409.

37. Mandala S, Hajdu R, Bergstrom J, Quackenbush E, Xie J, Milligan J, Thornton R, Shei G-J, Card D, Keohane CA, Rosenbach M, Hale J, Lynch CL, Rupprecht K, Parsons W, Rosen H (2002) Alteration of lymphocyte trafficking by sphingosine-1-phosphate receptor agonists. Science 296:346-349.

38. Brinkmann V, Davis MD, Heise CE, Albert R, Cottens S, Hof R, Bruns C, Prieschl E, Baumruker B, Hiestand P, Foster CA, Zollinger M, Lynch KR (2002) The immune modulator FTY720 targets sphingosine 1-phosphate receptors. J Biol Chem 277:21453-21457.

39. Adams DR, Tawati S, Berretta G, Rivas PL, Baiget J, Jiang Z, Alsfouk A, Mackay SP, Pyne NJ, Pyne S (2019) Topographical Mapping of Isoform-Selectivity Determinants for J-Channel -Binding Inhibitors of Sphingosine Kinases 1 and 2. J Med Chem 62:3658-3676.

40. Pitson SM, Moretti PA, Zebol JR, Lynn HE, Xia P, Vadas MA, Wattenberg BW (2003) Activation of sphingosine kinase 1 by ERK1/2-mediated phosphorylation. EMBO J 22:5491-5500

41. Pitson SM, Xia P, Leclercq TM, Moretti PA, Zebol JR, Lynn HE, Wattenberg BW, Vadas, MA (2005) Phosphorylation-dependent translocation of sphingosine kinase to the plasma membrane drives its oncogenic signaling. J Exp Med 201:49-54.

42. Inagaki Y, Li PY, Wada A, Mitsutake S, Igarashi Y (2003) Identification of functional nuclear export sequences in human sphingosine kinase 1. Biochem Biophys Res Commun 311:168-173.

43. Igarashi N, Okada T, Hayashi S, Fujita T, Jahangeer S, Nakamura S-I (2003) Sphingosine kinase 2 is a nuclear protein and inhibits DNA synthesis. J Biol Chem 278:46832-46839.

44. Wang Z, Min X, Xiao S-H, Johnstone S, Romanow W, Meininger D, Xu H, Liu J, Dai J, An S, Thibault S, Walker N (2013) Molecular basis of sphingosine kinase 1 substrate recognition and catalysis. Structure 21:798-809.

45. Gustin DJ, Li Y, Brown ML, Min X, Schmitt MJ, Wanska M, Wang X, Connors R, Johnstone S, Cardozo M, Cheng AC, Jeffries S, Franks B, Li S, Shen S, Wong M, Wesche H, Xu G, Carlson TG, Plant M, 
Morgenstern K, Rex K, Schmitt J, Coxon A, Walker N, Kayser F, Wang Z (2013) Structure guided design of a series of sphingosine kinase (SphK) inhibitors. Bioorg Med Chem Lett 23:4608-4616.

46. Wang J, Knapp S, Pyne NJ, Pyne S, Elkins JM (2014) Crystal Structure of Sphingosine Kinase 1 with PF-543. ACS Med Chem Lett 5:1329-1333.

47. Schwartz GK, Haimovitz-Friedman A, Dhupar SK, Ehleiter D, Maslak P, Lai L, Loganzo F, Kelsen DP, Fuks Z, Albino AP (1995) Potentiation of apoptosis by treatment with the protein kinase C-specific inhibitor safingol in mitomycin C-treated gastric cancer cells. J Natl Cancer Inst, 1995, 87, 1394-1399.

48. Sachs CW, Safa AR, Harrison SD, Fine RL (1995) Partial inhibition of multidrug resistance by safingol is independent of modulation of P-glycoprotein substrate activities and correlated with inhibition of protein kinase C. J Biol Chem 270:26639-26648.

49. Kedderis LB, Bozigian HP, Kleeman JM, Hall RL, Palmer TE, Harrison SD, Susick RL (1995) Toxicity of the protein kinase $\mathrm{C}$ inhibitor safingol administered alone and in combination with chemotherapeutic agents. Fundam Appl Toxicol 25:201-217.

50. Olivera A, Kohama T, Tu Z, Milstien S, Spiegel S (1998) Purification and characterization of rat kidney sphingosine kinase. J Biol Chem 273:12576-12583.

51. Högenauer K, Billich A, Pally C, Streiff M, Wagner T, Welzenbach K, Nussbaumer P (2008) Phosphorylation by sphingosine kinase 2 is essential for in vivo potency of FTY720 analogues. ChemMedChem 3:1027-1029.

52. Albert R, Hinterding K, Brinkmann V, Guerini D, Müller-Hartwieg C, Knecht H, Simeon C, Streiff M, Wagner T, Welzenbach K, Zécri F, Zollinger M, Cooke N, Francotte E (2005) Novel Immunomodulator FTY720 Is Phosphorylated in Rats and Humans To Form a Single Stereoisomer. Identification, Chemical Proof, and Biological Characterization of the Biologically Active Species and Its Enantiomer. J Med Chem 48:5373-5377. 


\section{Figure Legends}

Figure 1. Structures of sphingosine kinase substrates and inhibitors relevant to the analysis in this article. The canonical endogenous substrate for SK1 and SK2 is D-erythro-sphingosine (Sph), which is phosphorylated on the 1-OH to generate sphingosine-1-phosphate (S1P), a ligand that acts on the S1P family of GPCR receptors. Fingolimod (FTY720) is phosphorylated in vivo by SK2 on its pro-S hydroxymethyl arm to generate the immunomodulatory drug, (S)-FTY720-P [52]; for SK1, FTY720 is, at best, a very weak substrate that exhibits inhibitory activity $\left(K_{\mathrm{i}} 2 \mu \mathrm{M}\right)$ [23, 24]. ROMe is an SK2 selective inhibitor formally derived from FTY720 by methylation of the pro- $R$ hydroxymethyl (SK2 $K_{\mathrm{i}} 16 \mu \mathrm{M}$; SK1 no inhibition at 100 $\mu \mathrm{M})$ [26]. Co-crystal structures with SK1 have been obtained with three inhibitors to date: SKi (SK1 $K_{\mathrm{i}} 16$ $\mu \mathrm{M}$; SK2 $K_{\mathrm{i}} 6.7 \mu \mathrm{M}$ ) [44], Amgen-23 (SK1 IC 5020 nM; SK2 IC $501.6 \mu \mathrm{M}$ ) [45] and PF-543 (SK1 IC 5028 nM; SK2 IC $_{50}>5 \mu \mathrm{M}$ ) [39]. HWG-35D is a PF-543 analogue in which the selectivity has been switched from SK1 to SK2 by modification of the tail region (SK1 IC $5_{50} 4.1 \mu \mathrm{M}$; SK2 IC $5041 \mathrm{nM}$ ) [39]. Sphingadienes are naturally occurring sphingoid bases that are efficient substrates for both SK1 and SK2 (see data in Fig. 2). Safingol is an SK1- and PKC-inhibitory sphingoid base with anti-cancer activity (SK1 $K_{\mathrm{i}}$ ca. $5 \mu \mathrm{M}$ ) $[35,50]$ that retains appreciable substrate behaviour for SK2 (see data in Fig. 2).

\section{Figure 2. $h \mathrm{hK} 1$ and $h \mathrm{SK} 2$ use sphingadienes as a substrate, while safingol is a substrate for $h \mathrm{SK} 2$.}

Purified $h$ SK1 activity was assayed using $3 \mu \mathrm{M}$ Sph and $250 \mu \mathrm{M}$ ATP. Purified $h$ SK2 activity was assayed using $10 \mu \mathrm{M}$ Sph and $250 \mu \mathrm{M}$ ATP using an established isoform-specific radiometrc assays [29]. The substrate concentration of sphingosine corresponds to the $\mathrm{K}_{\mathrm{m}}$ of the enzymes, while the ATP concentration is saturating. Sphingadienes and safingol were used at the concentrations shown. SK activity is expressed as pmoles of phosphorylated product/min/ $\mu$ g enzyme. Assays were performed in triplicate.

Figure 3. Structure of $\boldsymbol{h S K 1} 1$ and detail of Sph substrate binding. (A) The structure of SK1 (PDB 3VZB) is shown with Sph (orange surface) bound in the C-terminal domain (CTD); Mg-ADP (green sphere/pink surface) is shown superimposed from a separate SK1 co-crystal (3VZD) to identify the position of the nucleotide binding site in the N-terminal domain (NTD) [44]. Three lipid binding loops (LBL-1 to LBL-3; 
cyan/salmon/yellow ribbon) fold across the $\beta$-sandwich core of the CTD to generate the lipid binding J-channel (mesh). A regulatory R-loop (geen ribbon) packs on the reverse face of the CTD $\beta$-sandwich. (B) Detail of the Sph binding site is shown extracted from panel (A). The Sph head group is orientated by a highly organised polar network of residues on a DGE-containing hairpin (141-DGE-143) together with an aspartate (Asp178) on LBL-1 and a structural water (W1) to present the Sph 1-OH to a second aspartate (Asp81) that is located on the phosphate-binding P-loop and that serves as the catalytic base during substrate turnover. The $\alpha$ - and $\beta$ phosphate groups of ATP, not shown in panel (B), are engaged by the enzyme's P-loop and T-loop respectively. Arginine residues (not shown) on the tip of LBL-1 are also thought to contribute to binding of ATP and likely orgnaise the $\gamma$-phosphate proximal to the Sph 1-OH [36]. The lipid head group environment is completed by the packing of Leu268 located on LBL-2. (C) A schematic representation of the SK1 J-channel and Sph engagement interactions is shown. Residues (Ile174, Met272 and Phe288) that differ in the J-channel surface of $h$ SK2 are marked (green text). These, and especially the replacement of Phe288 by cysteine in $h$ SK2, likely impact on isoform inhibitory selectivity for some synthetic inhibitor series (see text). Two other residue differences (Ala175/Ala339, replaced by Ser/Thr in SK2), marked in red in panel (B), are predicted to affect the packing of LBL-1 against the CTD $\beta$-sandwich core. These two residue differences also likely affect isoform selectivity for certain inhibitors and may additionally be important as determinants of substrate selectivity for SK1 and SK2.

Figure 4. Inhibitor binding interactions based on SK1 co-crystal structures and an SK2 homology model. (A) Ligand binding detail is shown schematically for the inhibitor, SKi, taken from its co-crystal structure (3VZC) with $h$ SK1 [44]. Key features of the J-channel and head group interaction network are marked, as described in the caption for Fig. 3. In the case of the SKi co-crystal structure, additional waters (W2, W3) are seen at the positions of the Sph 3-OH and 1-OH groups. (B) Corresponding detail is shown for the inhibitor, Amgen-23, taken from its co-crystal structure (4L02) with $h$ SK1 [45]. Here, the hydroxymethyl of the extended piperidine head group occupies the W2/Sph 3-OH site whilst the head group ring hydroxyl maps to the W3/Sph 1-OH site. (C) Protein engagement detail is shown for the inhibitor, PF-543, taken from its co-crystal structure (4V24) with hSK1 [46]. The interaction of the 2-(hydroxymethyl)pyrrolidinium head group of PF-543 is very similar to that of the piperidine-based head group of Amgen-23, but it lacks the ring hydroxyl engagement of 
the catalytic base residue (Asp81). Also lacking is a hydrogen bonded contact between PF-543 and the Jchannel threonine, Thr196, which is established with the aminothiazole subunits of both Amgen-23 and SKi. (D) A ligand interaction diagram is shown for the SK2-selective inhibitor, HWG-35D (compound 55 in Adams et al. [39]), docked into the published SK1-derived homology model of SK2 [39]. HWG-35D is a close analogue of PF543 but with reversed isoform selectivity. J-channel throat residues in hSK1 (Ile174, Met272) are replaced by Val304 and Leu517 in hSK2. However, the key J-channel differences that are thought to be responsible for the reversal of isoform selectivity are an expansion in the toe region (due to replacement of Phe288 in $h$ SK1 by Cys533 in hSK2) and a contraction in the heel due to inward movement of the LBL-3 heel residue (Leu547 in $h$ SK2) relative to that in $h$ SK1 (Leu302, marked in panel (A)).

Figure 5. Binding models for FTY720 and its methyl ether derivative, ROMe, with $h$ SK1 and $h S K 2$. (A) A docked model of FTY720 (orange stick) with SK1 is presented (binding mode 'a') in which the ligand's pro-S hydroxymethyl is presented to the catalytic base in a manner that might permit turnover to form (S)FTY720-P. Although this binding mode [boxed inset (i)], positions the ligand's $p$-phenylene subunit into the same space as defined for the $p$-phenylene of PF-543 in crystal structure 4V24 [boxed inset (ii) and overlay in (iii)], it requires an unfavourable backbone eclipsing interaction and would inolve suboptimal geometric engagement of the LBL-1 anchor residue, Asp308, thus explaining why FTY720 is not a substrate (or, at best, only a very weak substrate) for SK1. (B) An alternative docked model of FTY720 (orange stick) with SK1 is presented (binding mode 'b’). In this model the backbone torsions of FTY720 are relaxed and the head group rotated so that the pro-S hydroxymethyl engages Asp178 (instead of Asp81) and the pro-R hydroxymethyl and ammonium ion form a pincer on the carbonyl of Leu268; binding may additionally be supplemented by a salt bridge between the ligand's ammonium ion and Asp81. Binding mode ' $\mathrm{b}$ ', with the pro-S hydroxymethyl out of position for phosphorylation, may account for the SK1-inhibitory behaviour of FTY720. $($ C) FTY720 (orange stick) is shown docked into the homology model of hSK2 [39]. It is hypothesised that the replacement of Ala175 and Ala339 in hSK1, marked in panel (B), by Ser305 and Thr584 permits greater conformational freedom for the LBL-1 anchor residue in hSK2 (Asp308, corresponding to Asp178 in hSK1). FTY720 is then accommodated with the pro-S hydroxymethyl engaging the catalytic base (Asp211 in hSK2), consistent with the known stereoselectivity for the SK2-mediated phosphorylation reaction in generating (S)-FTY720-P in 
vivo [52]. (D) ROMe (green stick) is shown docked into the homology model of hSK2 [38]. Here, the conformational switching of the hSK2 LBL-1 anchor residue (Asp308) is again predicted to be pivotal for accommodation of the ligand, but with the methyl ether fitting a notch between Leu297 and Leu513 (marked) and forming a water-bridged contact to Asp211 (W3). The hydroxymethyl group, hydrogen bonded to Leu513, is out of position for phosphorylation, consistent with the behaviour of ROMe as an SK2-selective inhibitor with weak or null substrate turnover. This binding mode [boxed inset (i)] positions the ligand's p-phenylene subunit into the same space as defined for the $p$-phenylene of Amgen-23 in crystal structure 4L02 [boxed inset (ii) and overlay in (iii)]; the locked conformation of the hSK1 LBL-1 anchor residue (Asp178) disfavours binding of ROMe to this isoform.

Figure 6. Sphingosine kinase binding models for $(4 E, n Z)$-sphingadienes and safingol. (A) $h S K 1$-docked models of three sphingadienes are shown superimposed (ligand stick colour coding as specified in the boxed inset). The flexible alkylidene tail allows accommodation of all three shingoid bases in the J-channel (mesh) in a manner similar to that defined for Sph in its co-crystal structure (3VZB) with SK1. (B) A model of safingol (orange stick) docked into hSK1 is shown. This model invokes a rotation of the head group (relative to the position defined for bound Sph) to avoid an unfavourable steric compression of the (S)-configured 3carbinol against the side chain of Leu268. The adjustment moves the ammomium ion into a salt bridge position on Asp81 and the hydroxymethyl into a non-turnover, hydrogen bonded position on the Leu268 carbonyl. (C) Safingol (magenta stick) is shown docked into the homology model of hSK2 [39]. Here, the alternative (lifted) rotameric state for the LBL-1 anchor residue (Asp308) is predicted to accommodate the ligand with hydroxymethyl engagement on the catalytic base, consistent with the known turnover of safingol as a substrate for hSK2 (cf. Fig. 2). 
$\overbrace{\mathrm{N}_{\mathrm{N} \mathrm{H}_{2}}^{\mathrm{OH}}}^{\text {pro-s }}$

fingolimod (FTY720) (drawn as free base)

(R)-FTY720 methyl ether (ROMe)

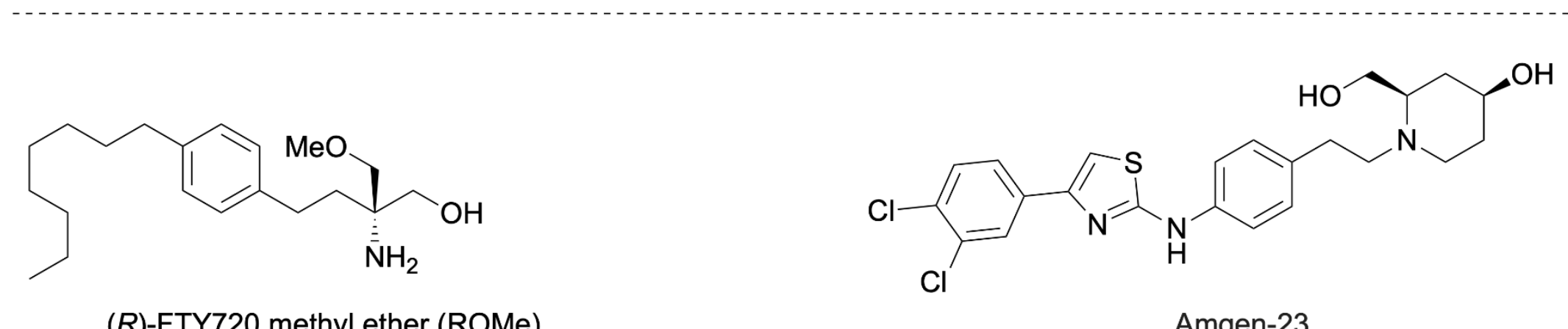

predominantly SK2<smiles>[R6]OC[C@@]([NH3+])(CO)CCc1ccc(CCCCCCCC)cc1</smiles>

fingolimod phosphate [(S)-FTY720P]

Amgen-23

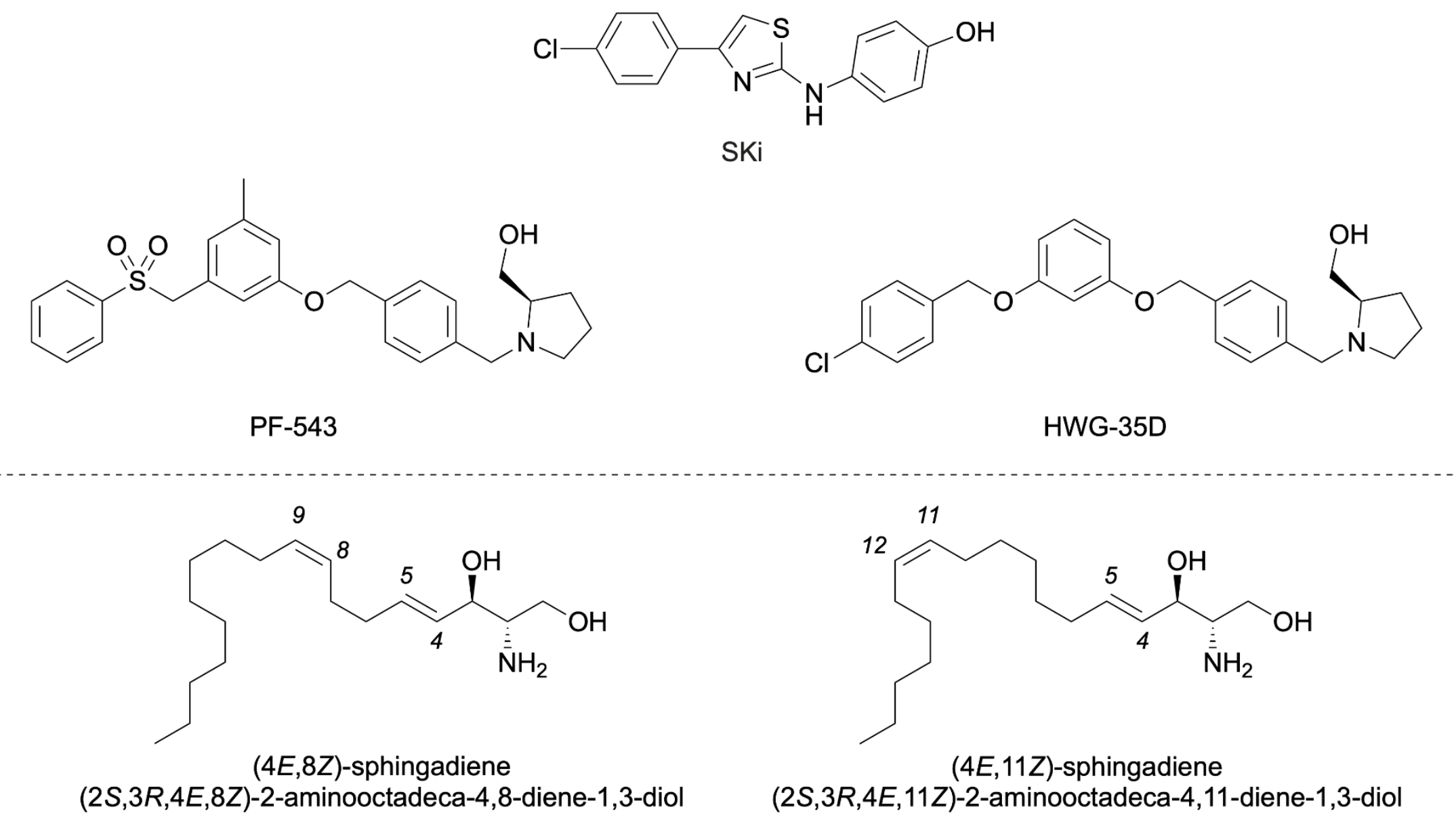

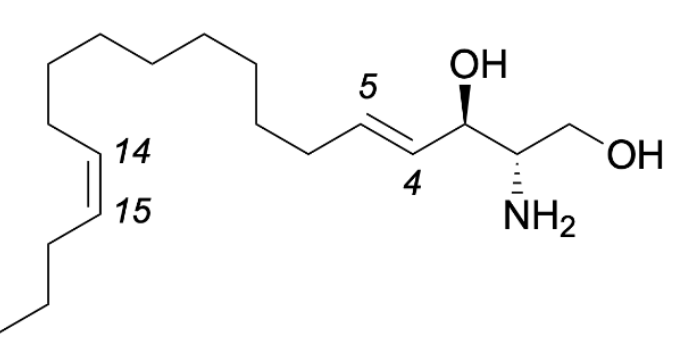

(4E,14Z)-sphingadiene

(2S,3R,4E,14Z)-2-aminooctadeca-4,14-diene-1,3-diol

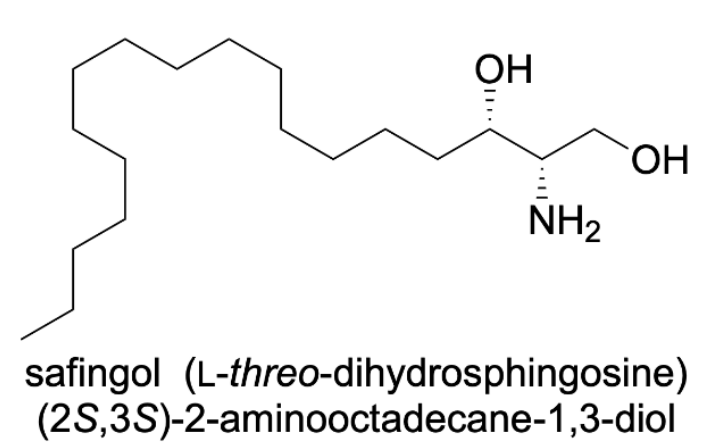


FIG 2
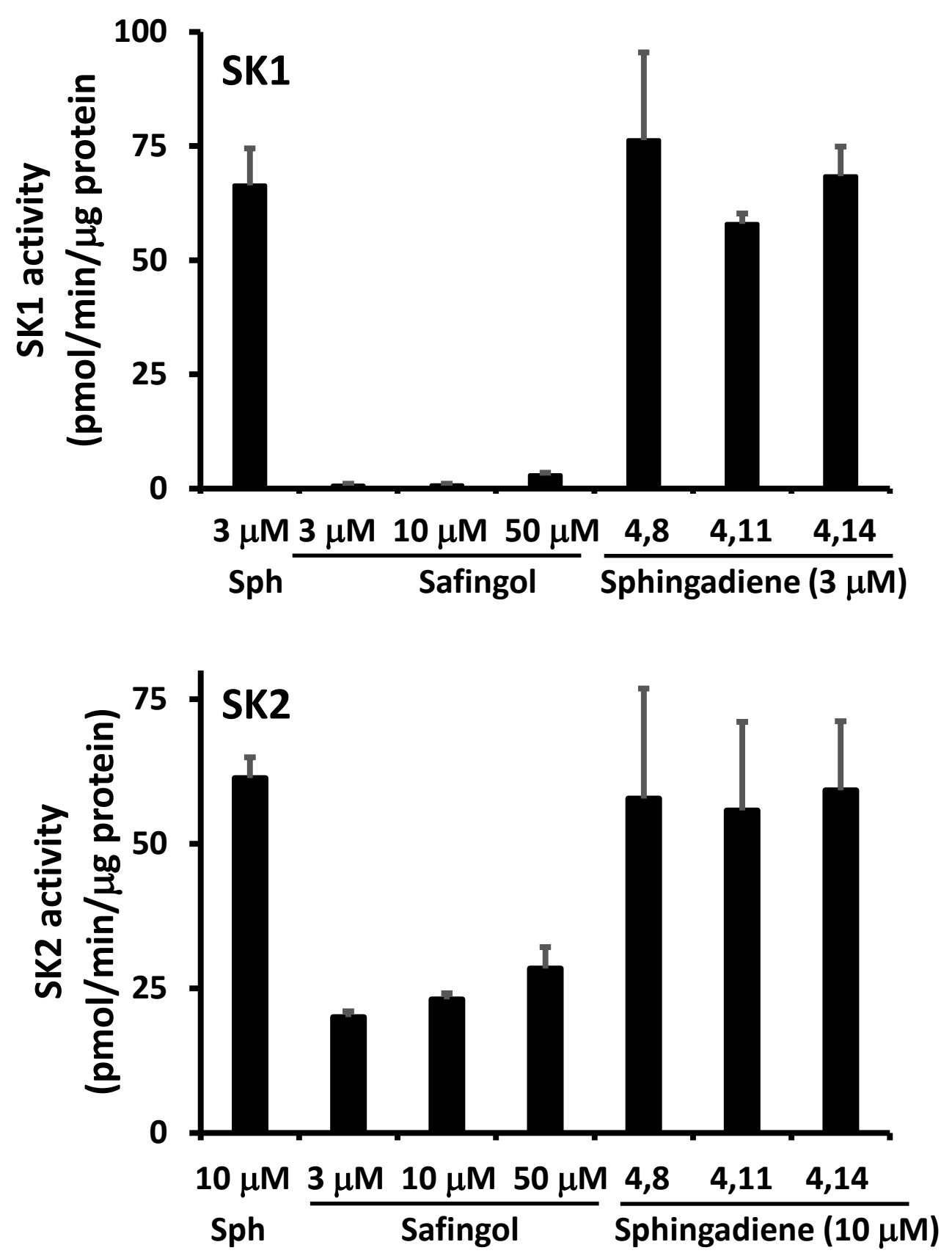
FIG 3

(A)
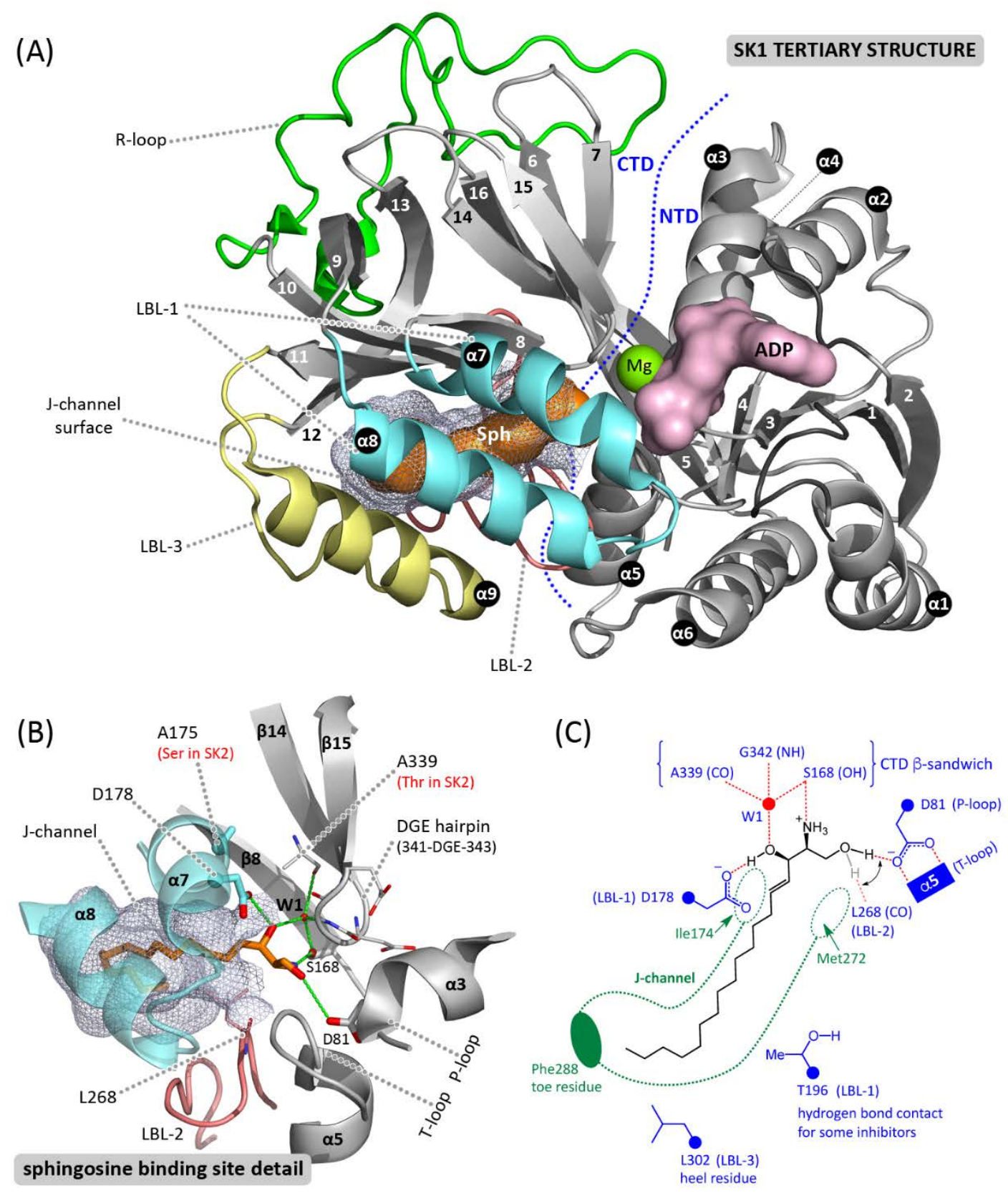


\section{(A) \\ SKi

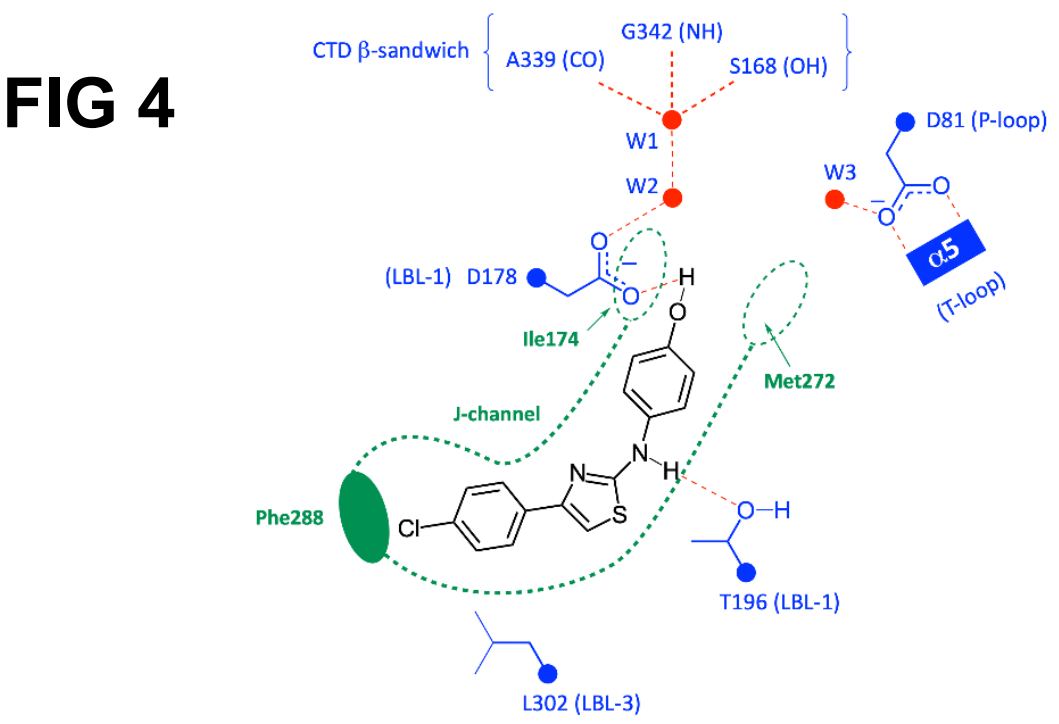

(C)

PF-543

crystallographic SK1 binding mode

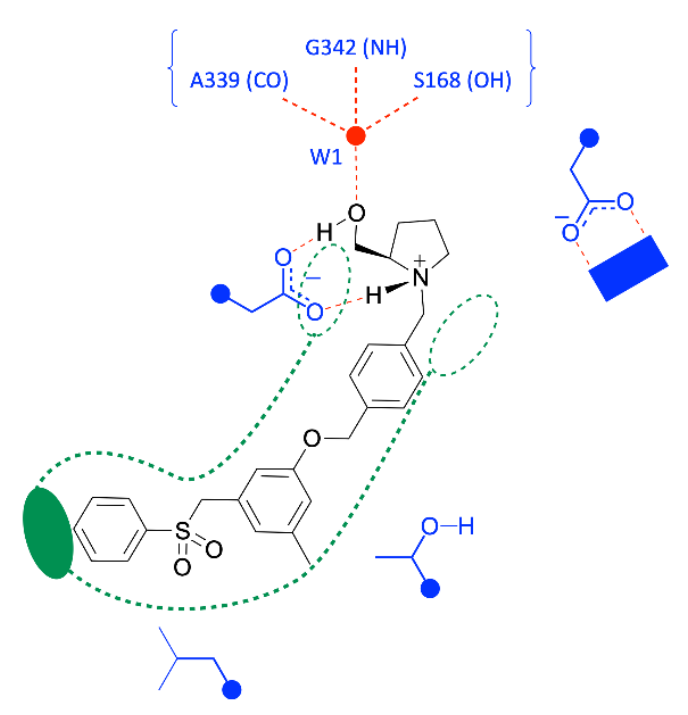

(B)

\section{Amgen-23}

crystallographic SK1 binding mode

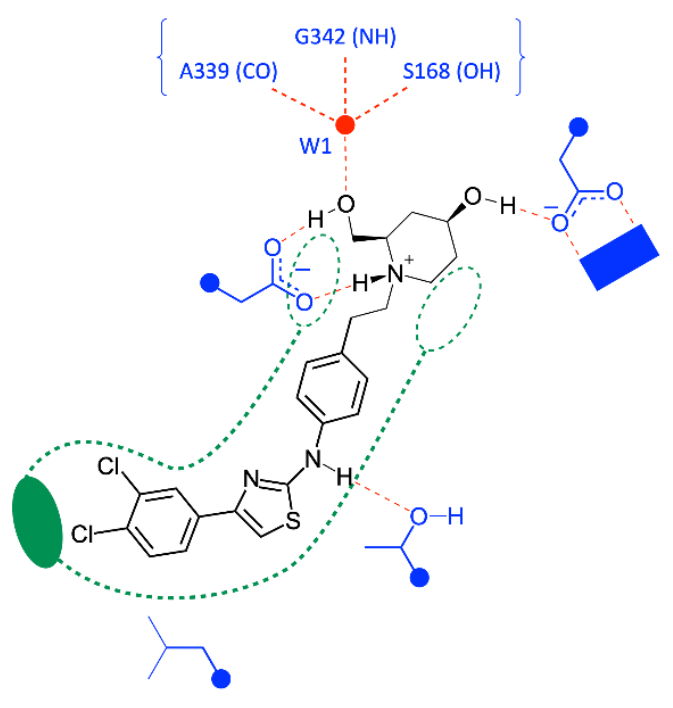

(D)

HWG-35D

SK2 binding model

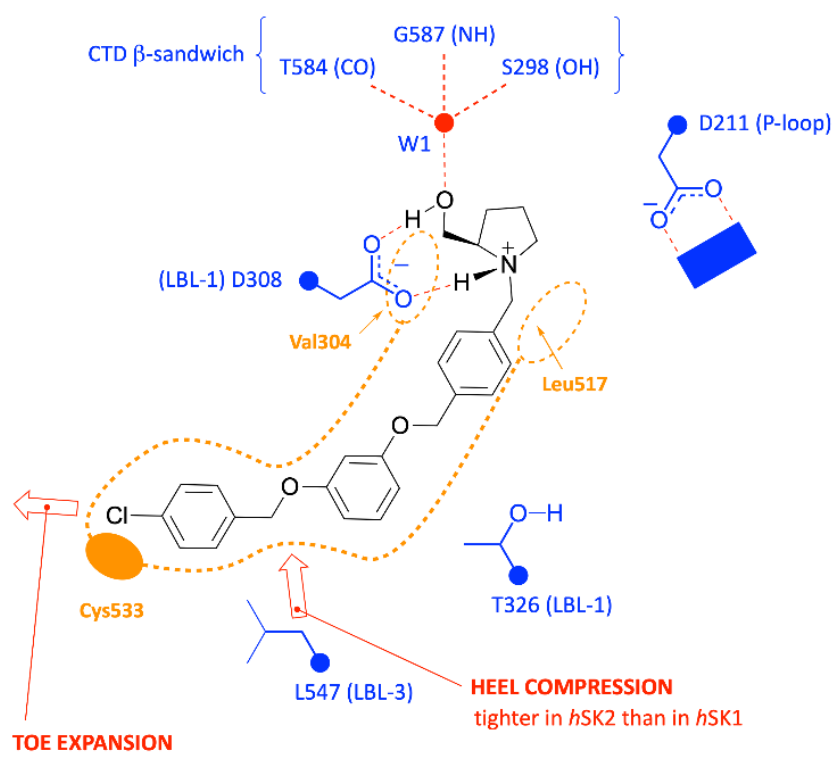




\section{FIG 6}

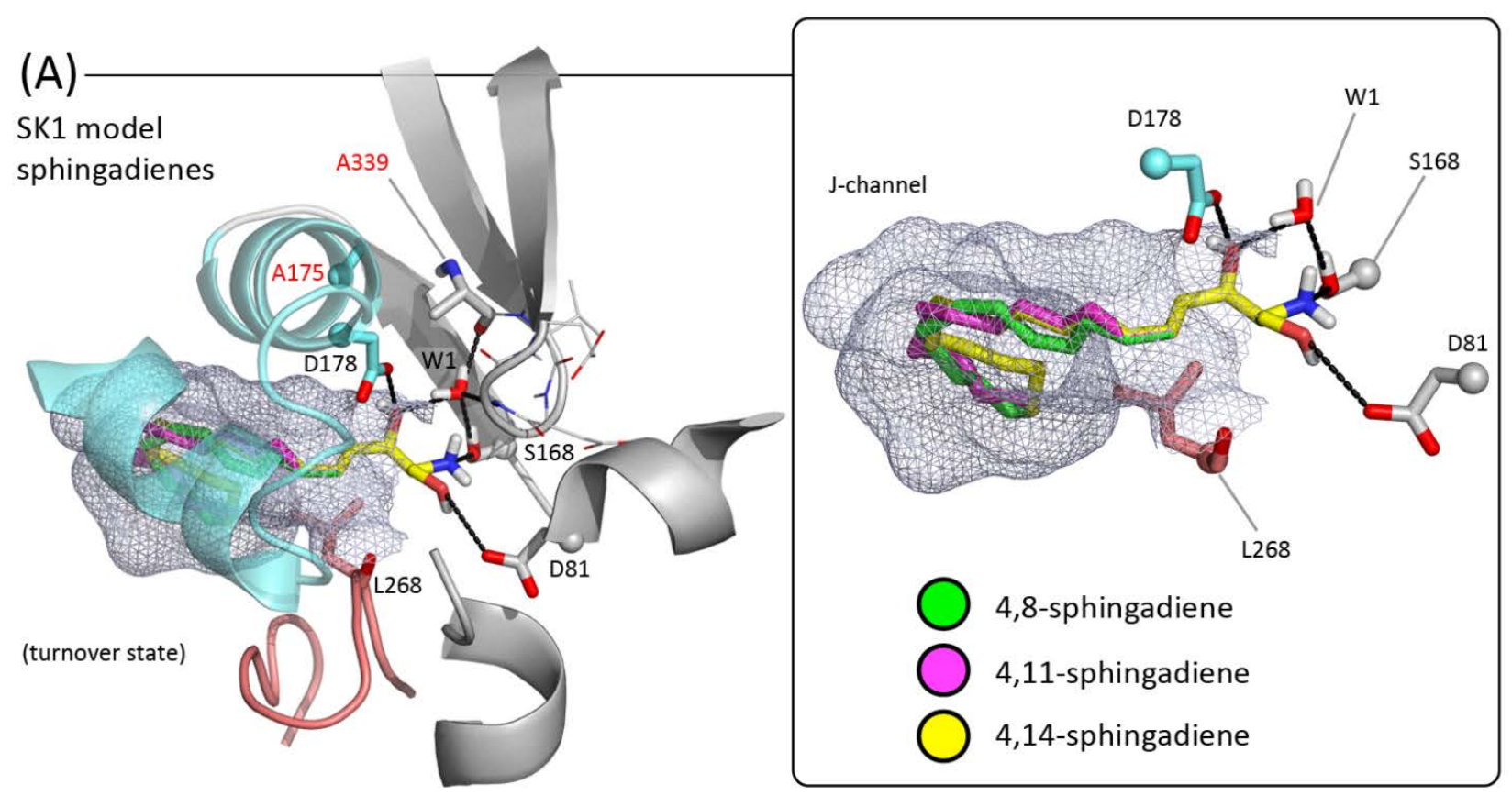

(B)

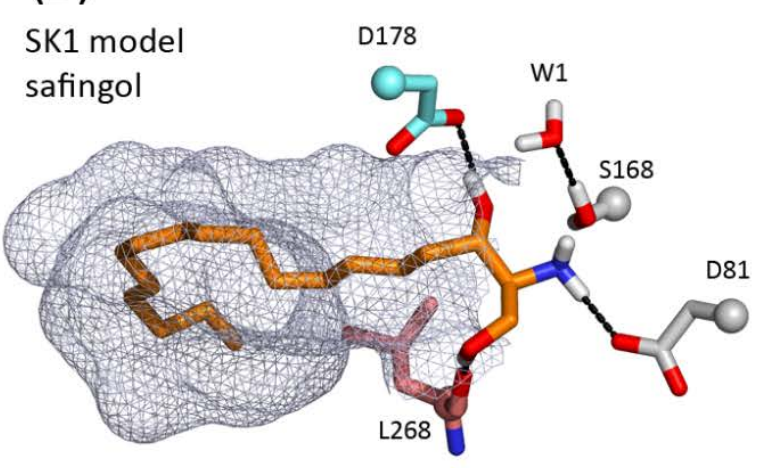

(non-turnover state)
(C)

SK2 model

safingol

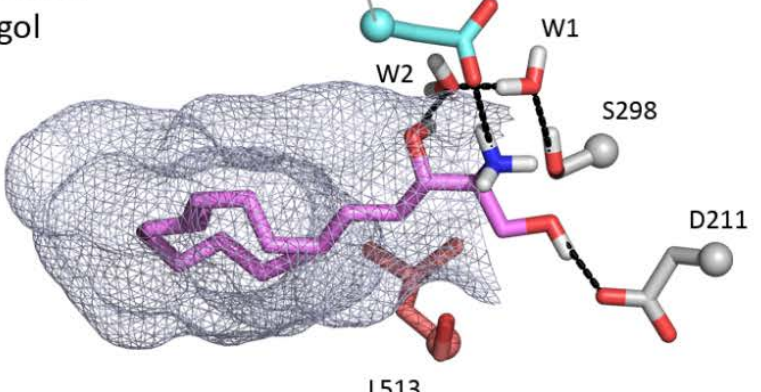

(turnover state)
D308 (alternative rotameric state)

L513 